\title{
Gas Mixing System for Imaging of Nanomaterials under Dynamic Environments by Environmental Transmission Electron Microscopy
}

\author{
Cem Akatay ${ }^{1}$, Yury Zvienevich ${ }^{2}$, Eric A. Stach ${ }^{3}$ and Fabio Ribeiro ${ }^{2}$ \\ 1. School of Materials Engineering and Birck Nanotechnology Center, Purdue University, West \\ Lafayette, Indiana 47907, USA \\ 2. Forney Hall of Chemical Engineering, Purdue University, West Lafayette, Indiana, USA \\ 3. Center for Functional Nanomaterials, Brookhaven National Laboratory, Upton, New York 11973, USA
}

The development of differentially pumped environmental cells for transmission electron microscopes and the use of heating holders has allowed researchers to create a reactive environment within the microscope that broadly approaches the working conditions of catalysts, while maintaining the ability to perform high resolution imaging, diffraction and spectroscopy. However many reactions involve the use of multiple gases where the composition of the gas mixture is dictated by the specifics of the reaction. Further, effective investigation of the dynamic behavior of materials necessitates the ability to vary the composition of individual gases in a mixture and also to switch from one specific environment to another with operational ease and imaging stability. To this purpose, we have developed a gas mixing manifold system that is capable of delivering a stable pressure stream of a desired composition of gases into an Environmental Transmission Electron Microscope (ETEM).

The gas manifold system is designed as a cart for easy handling and transportation. The system is equipped with seven mass flow controllers and is controlled by a mass flow \& pressure programmer. The performance of the system is evaluated by pressure, temperature fluctuations and sample drift during induced gas mixture composition changes. The variation of sample temperature under different pressure perturbations is shown in Figure 1. For the case of CO mixing, the incoming gas mixture composition is changed while the pressure of the system is kept constant. The temperature fluctuation for this compositional change is negligible and do not cause any significant sample drift as plotted in Figure 2 .

A mixture of up to six different gases can be introduced into the microscope without causing significant sample drift or temperature/pressure fluctuation.

We acknowledge support from the Institute for Atom-efficient Chemical Transformations (IACT), an Energy Frontier Research Center funded by the U.S. Department of Energy, Office of Science, Office of Basic Energy Sciences. E.S. acknowledges additional support from the Center for Functional Nanomaterials, Brookhaven National Laboratory, which is supported by the U.S. Department of Energy, Office of Basic Energy Sciences, under Contract No. DE-AC02-98CH10886. We thank to Dr. Dmitri Zakharov for his assistance at the Birck Nanotechnology Center. 


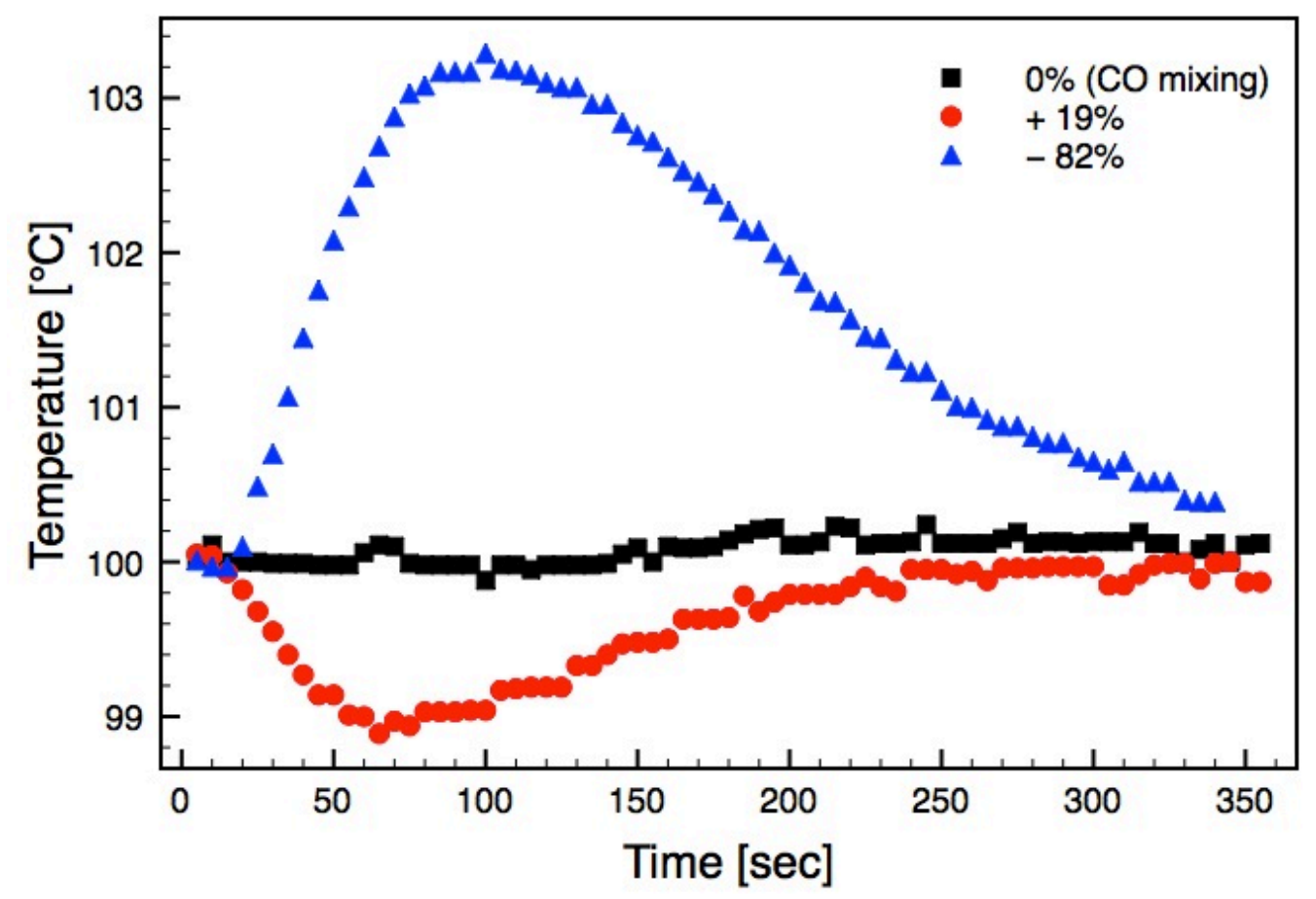

Figure 1. Sample temperature after different perturbations: CO introduction under constant pressure (squares), 19\% increase (circles), and 82\% decrease (triangles) in pressure

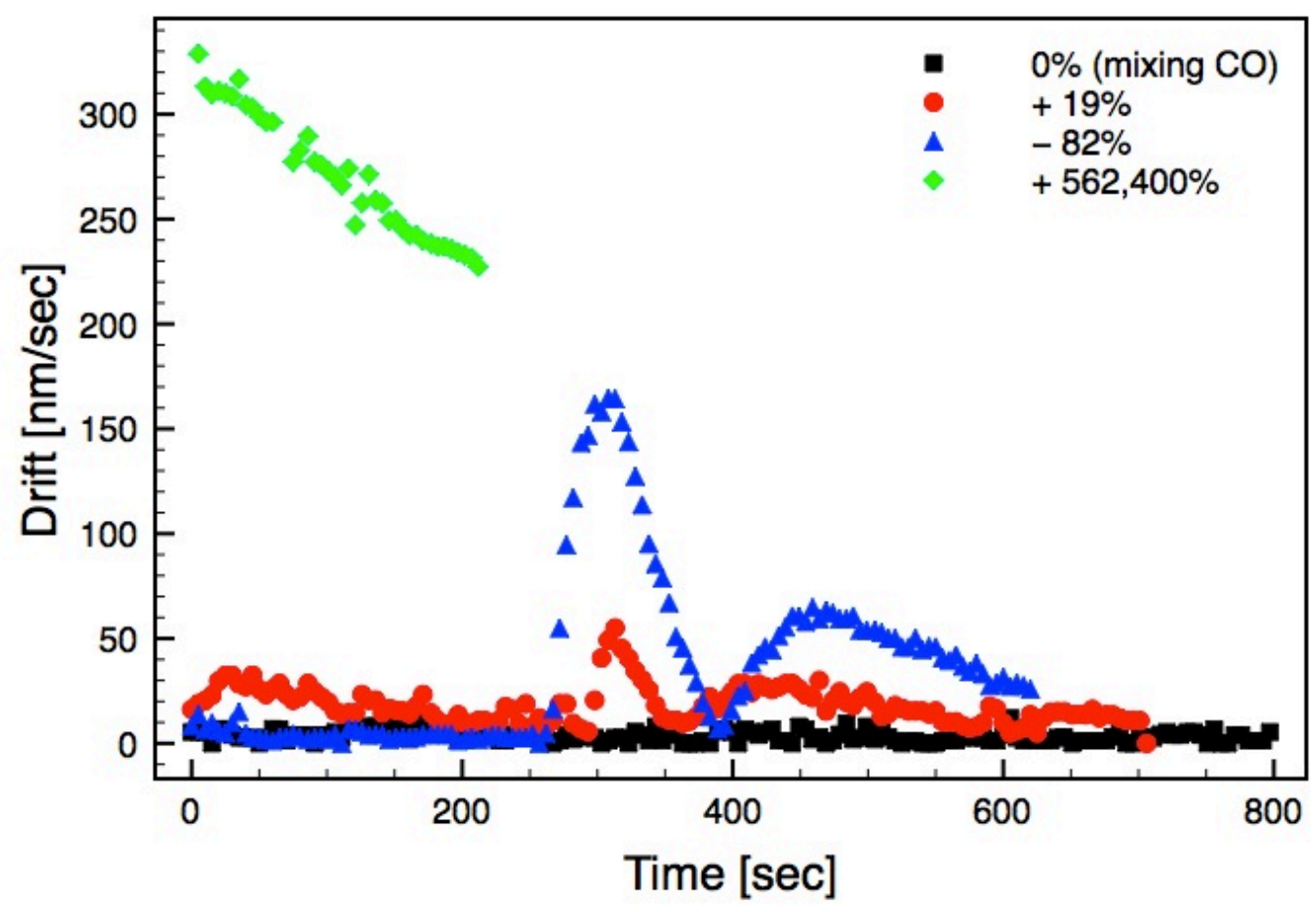

Figure 2. Amount of sample drift after different perturbations: $\mathrm{CO}$ introduction under constant pressure (square), 19\% increase (circle), 82\% decrease (triangle), and 562,400\% increase (diamond) in pressure 